Cahiers $d u$ MONDE RUSSE

\section{Cahiers du monde russe}

Russie - Empire russe - Union soviétique et États indépendants

$50 / 4 \mid 2009$

Varia

\title{
Boris B. Gorshkov, Russia's Factory Children
}

\section{Michel Tissier}

\section{OpenEdition \\ Journals}

Édition électronique

URL : https://journals.openedition.org/monderusse/7150

DOI : $10.4000 /$ monderusse. 7150

ISSN : $1777-5388$

\section{Éditeur}

Éditions de l'EHESS

\section{Édition imprimée}

Date de publication : 15 décembre 2009

Pagination : 781-785

ISBN : 978-2-7132-2261-0

ISSN : $1252-6576$

\section{Référence électronique}

Michel Tissier, «Boris B. Gorshkov, Russia's Factory Children », Cahiers du monde russe [En ligne], 50/4 | 2009, mis en ligne le 12 janvier 2011, consulté le 03 septembre 2022. URL : http:// journals.openedition.org/monderusse/7150; DOI : https://doi.org/10.4000/monderusse.7150

Ce document a été généré automatiquement le 3 septembre 2022.

Tous droits réservés 


\title{
Boris B. Gorshkov, Russia's Factory Children
}

\author{
Michel Tissier
}

\section{RÉFÉRENCE}

Boris B. GORSHKOV, Russia's Factory Children. State, Society, and Law, 1800-1917.

Pittsburgh : The University of Pittsburgh Press, 2009, XIII+216 p.

1 L'ouvrage de Boris B. Gorshkov est bref, mais non dénué d'ambitions. La première de ces ambitions consiste à combler une lacune dans l'historiographie de la Russie, notamment si on la compare avec la littérature historique, déjà bien fournie, sur le travail des enfants dans les grands pays industrialisés du XIX ${ }^{\mathrm{e}}$ siècle, en Europe et en Amérique du Nord. Le faible intérêt suscité jusqu'ici par la question du travail des enfants dans la Russie présoviétique ne laisse pas d'étonner, compte tenu de la richesse des études d'histoire sociale consacrées à ce pays et, surtout, de la portée idéologique qui a longtemps été la leur. C'est aujourd'hui dans un autre contexte et à partir d'autres prémisses que Gorshkov traite la question du travail des enfants à l'époque de l'industrialisation de la Russie. Et, justement, la lecture de son ouvrage laisse penser qu'une autre de ses ambitions consiste à dépolitiser les études d'histoire sociale.

2 Le livre est divisé en quatre chapitres dont les intitulés thématiques masquent un peu le découpage chronologique de l'ouvrage en trois moments principaux correspondant à la période préindustrielle et aux débuts de l'industrialisation (ch. I), au développement de l'industrie mécanisée entre les années 1840 et le début des années 1880 (ch. II et III) et, enfin, à la situation de la Russie, puissance industrielle en plein essor à partir de la fin du $\mathrm{XIX}^{\mathrm{e}}$ siècle (ch. IV). Le premier chapitre, sur «les origines du travail industriel des enfants ", présente les tenants du problème initial, en montrant ce que pouvaient signifier, dans la Russie préindustrielle, à la fois la catégorie de l'enfance et l'implication des enfants dans l'activité productive. Pour la période centrale, les chapitres II et III abordent successivement la place des enfants dans l'évolution socioéconomique 
enclenchée avec l'industrialisation de la Russie (« Les enfants dans l'industrie : contexte démographique et social ») et les retentissements de cette évolution sur la façon dont étaient perçus l'enfance et le travail des enfants dans les milieux éduqués (« Débats publics et efforts législatifs»). Enfin le dernier chapitre («Les enfants de l'usine: politique, éducation et État ») présente l'aboutissement des débats antérieurs sous la forme du décret de 1882, première mesure d'ensemble sur le travail des enfants dans l'industrie, et dans ses développements législatifs ultérieurs. Cette dernière section analyse également les conséquences de la nouvelle réglementation à la fois sur les entreprises privées et sur les conditions dans lesquelles les enfants ouvriers vivaient, travaillaient et grandissaient à la fin de la période impériale.

3 L'auteur situe ainsi dans la longue durée la constitution d'une législation spécifique sur l'emploi des enfants par le secteur industriel en plein essor en Russie au xix ${ }^{e}$ siècle. Il met en valeur une évolution fondamentale dans les conceptions relatives à l'enfance et au travail dans le contexte russe. Ce que le livre montre de façon particulièrement nette, c'est, d'une part, la façon dont la participation des enfants aux activités de production économique commença seulement au XIX ${ }^{e}$ siècle à être conçue comme un travail (p. 25); et, d'autre part, la façon dont ce travail vint à être reconnu au cours du siècle comme inadapté à la vocation de l'enfance, et même comme une pratique immorale eu égard à cette vocation.

4 En effet, le travail des enfants, tel qu'on peut l'envisager à partir du titre du livre - celui des «enfants de l'usine»-, correspondait en fait à un schéma nouveau au XIX ${ }^{\mathrm{e}}$ siècle. Celui-ci fut créé progressivement par les conditions de l'industrialisation en Russie, rompant avec la situation initiale. Dans la Russie préindustrielle, et encore durant les premiers temps de l'industrialisation, la participation des enfants aux activités productives répondait essentiellement à une logique éducative, c'est-à-dire à une forme d'apprentissage. Dans ce schéma ancien, dont on retrouve les traces dans la réglementation impériale dès la fin $\mathrm{du} \mathrm{XVIII}{ }^{\mathrm{e}}$ siècle, les enfants pouvaient être différenciés selon leur âge, leur sexe et leur force, ou leur capacité à effectuer telle ou telle tâche. Pour les adultes, l'implication des enfants permettait leur initiation aux tâches productives et leur préparation à la vie adulte, qu'il s'agisse des travaux des champs, du travail dans les manufactures des propriétaires fonciers ou de l'État, puis, bientôt, de la production dans les premières usines mécanisées des débuts de l'industrialisation.

5 Même si on ne peut pas dire qu'il y eut une hausse dans la proportion des enfants « au travail » par rapport à la période antérieure, l'auteur expose la transformation qui a affecté les représentations du travail et de l'enfance au cours du xix siècle. Ce changement est incontestablement lié au recours massif à la main-d'œuvre enfantine, et surtout juvénile, dans les nouvelles industries en pleine croissance. Après l'abolition du servage en 1861, la misère dans les campagnes et la croissance démographique poussèrent de nombreux paysans, adultes et enfants les plus âgés, à migrer vers les centres industriels. Les usines embauchaient les enfants pour effectuer quantité de tâches auxiliaires, peu ou pas rémunérées, en soutien au travail des adultes et souvent avec les mêmes horaires qu'eux. Dans ce contexte, la dimension formatrice du travail des enfants parut à nombre d'observateurs de plus en plus effacée par l'organisation de la production dans les nouveaux établissements industriels, qui avaient là une main-d'œuvre non qualifiée très bon marché.

6 L'examen de cette évolution permet à Gorshkov de déployer une autre ambition fondamentale de l'ouvrage: mettre en évidence l'ampleur des interactions à l'œuvre 
dans le processus de création du droit dans l'Empire russe. Selon l'auteur, les lois émergeaient de la discussion publique et du compromis entre différents groupes sociaux. L'historien montre en l'espèce combien l'administration tsariste joua un rôle moteur dans l'organisation des débats sur la question du travail des enfants, combien elle s'efforça de consulter les différentes parties en présence et d'aboutir à un accord sur la nécessité de la réforme, d'une part, et sur ses modalités d'application, d'autre part.

7 En l'occurrence, pour rendre compte du changement de perceptions sur le travail des enfants et du long processus qui mena à la première réglementation en la matière, l'auteur différencie deux moments successifs (distingués principalement dans le ch. III). Dans la première période, c'est-à-dire depuis les premières tentatives de réglementation partielle dans les années 1830 jusqu'aux années 1860 , le travail des enfants dans les établissements industriels commença à faire l'objet d'une confrontation entre deux types d'acteurs seulement. D'un côté se trouvaient les autorités gouvernementales et administratives qui, au niveau central et à l'échelle locale et provinciale, critiquaient de plus en plus les conditions dans lesquelles les industriels faisaient travailler les enfants. De l'autre côté se tenaient précisément les industriels et leurs représentants au sein d'associations qui exprimaient les intérêts des milieux d'affaires russes, bien que ces associations fussent à l'époque officiellement placées sous la tutelle de l'administration.

Gorshkov montre que, durant cette période, l'impulsion en faveur d'un changement d'attitude à l'égard du travail des enfants venait exclusivement des milieux officiels, de l'intérieur de la machine administrative tsariste, les responsables ministériels prenant le relais des responsables locaux et provinciaux. On ne peut pas dire que, en regard, les industriels tenaient le beau rôle. Leurs interventions consistaient essentiellement à refuser les mesures proposées pour éloigner les enfants des usines, où leurs conditions de travail et de vie étaient de moins en moins perçues comme appropriées par les officiels. Les employeurs expliquaient de leur côté qu'il valait mieux pour les enfants rapporter de l'argent à la famille et travailler dans des usines bien aérées plutôt que de croupir dans des maisons insalubres. Les autorités accueillaient ces arguments avec scepticisme, plusieurs officiels cités par l'auteur considérant ouvertement que les patrons recherchaient d'abord le profit et exploitaient les enfants des ouvriers sans vergogne.

Après les réformes des années 1860 , s'ouvrit une seconde période où intervinrent de nouveaux acteurs, qui renforcèrent le camp de la réforme contre les industriels. Il s'agissait des représentants élus des nouvelles institutions au niveau local, les zemstva et les doumas urbaines, auxquels s'ajoutaient de plus en plus les interventions de la presse périodique, sans oublier les ouvriers eux-mêmes à travers leurs diverses formes d'organisation, plus ou moins durables (manifestations, grèves, syndicats d'abord clandestins, puis autorisés après 1905). En face, les industriels continuaient majoritairement à s'opposer aux réformes projetées. Gorshkov peut ainsi contester de façon convaincante l'idée d'un patronat impuissant face à un État autocratique omnipotent. Il souligne la capacité des industriels à s'organiser et à défendre leurs intérêts collectivement. Le livre étudie en détail les différentes commissions constituées par l'administration, depuis les années 1830 jusqu'au début des années 1880, pour proposer la restriction et le contrôle du travail des enfants et, parallèlement, pour inciter ou obliger les industriels à prendre en charge leur éducation scolaire. Ces propositions, longuement débattues par les différentes parties, ne débouchèrent pas en raison de l'opposition des industriels. Ce n'est qu'avec l'adoption du décret de 1882 que fut réglementé pour la première fois de façon globale le travail des enfants dans l'industrie. 
Surtout, cette législation permit de créer les conditions d'un contrôle effectif des usines grâce à la création d'un corps d'inspecteurs qui réussit à s'implanter, supervisa les relations entre patrons et ouvriers (enfants, puis également adultes), et constitua aussi de précieuses sources d'informations statistiques sur l'économie industrielle en Russie.

L'auteur prolonge son raisonnement en montrant que ces avancées sur la réglementation du travail des enfants servirent de matrice pour le développement de la législation sur le travail dans son ensemble à la fin de la période impériale, dans une logique tout à fait comparable à ce que l'on retrouve pour les autres pays industrialisés d'Europe à la même époque. Gorshkov en profite pour souligner le fait que l'administration impériale russe était loin d'être en retard, ou "arriérée ", dans son approche des questions relatives au travail des enfants et de la législation sur le travail en général. De ce point de vue, il montre que la chronologie des réformes en Russie est tout à fait parallèle à celle des autres pays industrialisés européens (p. 144-146). Le chapitre IV note néanmoins que le déclenchement de la Grande Guerre marqua le début d'une période de retour en arrière $\mathrm{du}$ point de vue de la législation sur le travail en général, et en particulier pour les enfants. Mais, là encore, l'argument de l'auteur selon lequel la Russie s'inscrit dans une évolution commune à l'échelle européenne est convaincant.

Cependant, dans son ambition d'éclairer sous un jour nouveau la fabrication du droit sous le régime impérial, l'auteur analyse l'impulsion réformatrice de l'administration et l'influence parallèle des industriels d'une façon qui peut laisser le lecteur sur sa faim. C'est une chose de vouloir dépolitiser le sujet abordé, et il est vrai que, dans l'argumentation des industriels en faveur du statu quo, leur hypocrisie parle d'ellemême, sans que l'auteur ait besoin d'en rajouter. Encore faut-il expliquer toute la difficulté que l'administration eut à faire passer les réformes proposées dans les années 1870, 1860 et encore avant. L'auteur montre lui-même, en s'appuyant sur les témoignages des contemporains (p.136-137), combien les avancées législatives furent en dernière instance le produit des circonstances économiques et de la crise du tournant des années 1880, qui provoqua faillites et chômage et fit taire momentanément les critiques des industriels. Comment expliquer alors l'incapacité de l'administration, " en temps normal ", à faire prévaloir ses vues réformatrices ? D’où venait la force de conviction des industriels? De la pertinence de leurs arguments sur les désavantages des restrictions au travail des enfants, dans la concurrence internationale à laquelle les entreprises russes étaient soumises? Ou d'autres éléments doivent-ils être pris en compte, qui permettraient de mieux saisir les ressorts de leur influence? L'auteur cite bien, à quelques moments, les réticences de certains milieux gouvernementaux, notamment au sein du ministère des Affaires intérieures, à l'égard des projets de réglementation. Mais il ne pousse pas l'analyse plus loin. De même la caractérisation comme "sphère publique ", selon la terminologie empruntée à Habermas, du fonctionnement des groupes de pression industriels dans leur relation à l'administration, ne suffit pas à rendre compte de l'influence de ces industriels.

12 C'est une question importante que l'ouvrage de Gorshkov invite à creuser. Mais son texte dense propose quantité d'autres pistes intéressantes à suivre, depuis l'exploitation des informations recueillies par le corps des inspecteurs des usines, qu'il serait utile de compléter par une étude du personnel des inspecteurs, jusqu’à la question, soulevée dans le dernier chapitre, de l'implication des enfants ouvriers dans les développements politiques du début du $\mathrm{xx}^{\mathrm{e}}$ siècle. Il faut savoir gré à l'auteur d'avoir su fournir autant de matière à exploiter dans un ouvrage aux dimensions aussi modestes. 